\title{
Persistent ulceration of the oral floor: a case of necrotizing sialometaplasia of the sublingual gland?
}

\author{
Cédric Alande*, Mathilde Fénélon, Sylvain Catros, Jean-Christophe Fricain \\ Odontology Department, Hôpital Pellegrin, Bordeaux, France \\ *Correspondence: cedric.alande@laposte.net
}

(Received: 1 August 2017, accepted: 2 August 2017)

Keywords:

necrotizing sialometaplasia /

sublingual gland / ulceration

\begin{abstract}
Introduction: Necrotizing sialometaplasia is an inflammatory, rare and benign pathology, affecting particularly minor salivary glands. Observations: A case of deep and persistent ulceration of the oral floor associated with sensitivity disorders is reported. Three biopsies were realized, each concluding to a nonspecific ulceration. Local treatments by triamcinolone injections and laser $\mathrm{CO}_{2}$ were tested. After 18 months of follow-up, a partial regression of the lesion was observed and the patient did not have any sensitivity disorders. Comments: This ulceration was clinically evocative of malignant neoplasia, which was reversed by histology. 0thers causes of ulceration were excluded on clinical and biological arguments. By elimination, a diagnosis of necrotizing sialometaplasia of the sublingual gland was made. Conclusion: Only one case of necrotizing sialometaplasia of sublingual gland has already been published. The case described here is exceptional due to the low prevalence of the lesion and the chronic ulcer evolution.
\end{abstract}

\section{Case study}

In October 2015, a 29-year-old patient consulted the oral surgery department for a deep ulcer on the floor of the mouth that had developed over 4 months. The patient reported a rapid increase in lesion size. The clinical examination revealed an elevated necrotic crater-shaped ulceration. The ulceration was approximately $3 \mathrm{~cm}$ diameter. A peripheral erythematous halo was observed (Fig. 1). The lesion was painful and accompanied by a numbness of the tongue tip. At the end of August 2015, a biopsy had been performed. Histological analysis revealed a nonspecific, fleshy, bud-like granulated tissue with ulceration. Following this first consultation, a Treponema pallidum hemagglutination assay - venereal disease research laboratory (TPHA-VDRL) and HIV 1 and 2 antibody tests were ordered, as well as a complete blood count with serum electrophoresis. All of the laboratory test results were strictly normal. A magnetic resonance imaging scan and cervicofacial computed tomography scan were performed and did not confirm any diagnosis. The ulceration at first appeared to be a case of malignant neoplasia. A second biopsy was performed which indicated a polymorphic inflammatory ulceration. Through process of elimination, a diagnosis of necrotizing sialometaplasia (NS) of the sublingual gland was made. However, the lesion did not spontaneously heal, local treatment with three injections of triamcinolone (Kénacort $80 \mathrm{mg}$ ) spaced 3 weeks apart was administered between December 2015 and January 2016. The patient then reported an alleviation of pain. Clinically, we observed that the inflammation of the lesion had decreased and the lesion was less indurated on palpation. However, the necrotic background persisted with an overall a loss of matrix. In February 2016, a $\mathrm{CO}_{2}$ laser treatment was initiated without any significant improvement. In March 2016, a surgical reconstruction had been planned, before it was abandoned because of the onset of scarring. After 11 months (June 2016), we observed a partial regression of the lesion and an improvement in tongue sensation. After 18 months (November 2016), tongue sensation had been completely restored, but the healing process seemed to have halted (Fig. 2). A final biopsy was performed in February 2017 to try to clarify the diagnosis. It confirmed the histological diagnosis of fleshy bud granulated tissue. The patient was reviewed in June 2017, the lesion was asymptomatic and had decreased in size.

\section{Comments}

Described for the first time in 1973 by Abrams et al., NS is a benign inflammatory pathology of the salivary glands, whose etiopathogenesis is still poorly defined [1,2]. Rare cases of NS of the salivary glands have been reported, one of which can affect the sublingual gland [3]. The diagnosis of the case reported here is of particular interest, because of the chronic nature of the ulceration. This case was a severe example and could be potentially misdiagnosed as malignant neoplasia. The histological analysis confirmed that it was not malignant neoplasia. The clinical and histological aspect of NS can often be misdiagnosed as a carcinogenic lesion, which can sometimes lead to unnecessary surgical intervention [4]. Here the pathological tests did not confirm the diagnosis, this could be because of the fact that the biopsies were performed after 


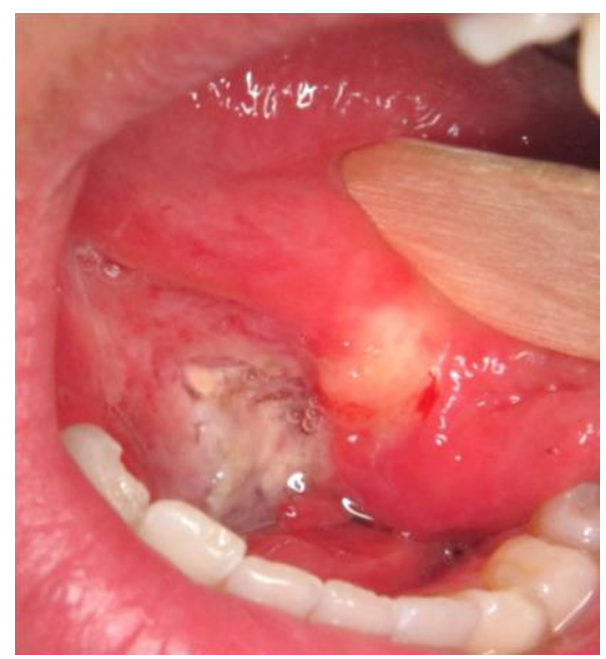

Fig. 1. Aspect of the lesion in 0ctober 2015 (intraoral photography).

the onset of the ulceration which could explain the appearance of fleshy bud-like granulated tissue. The various causes of chronic ulceration found in the literature may be eliminated on clinical or biological grounds such as traumatic, infectious (syphilis, tuberculosis, CMV, herpes, HIV, histoplasmosis, mucormycosis), autoimmune (pemphigoid, lichen planus), toxic epidermal necrolysis, drug intake, eosinophilic ulcers. By elimination, the only diagnosis compatible with the clinical history was NS of the sublingual gland, because of the appearance of ulceration and its unilateral localization.

Although an ischemic event preceding lesion onset is the most commonly accepted cause of this condition, the etiopathogenesis of NS remains controversial. NS often heals on its own, eventually fading away to form a depressed scar. The healing time is 3-5 weeks depending on the initial size of the lesion $[2,3]$. Regular monitoring should be conducted afterward. Other treatments are mentioned in the literature: intralesional injection of corticosteroids [3], laser, and surgical resection. The first two treatments can be administered without completely scarring the ulceration. Regular monitoring was conducted because of the absence of any pain or discomfort and the failure of the treatments attempted.

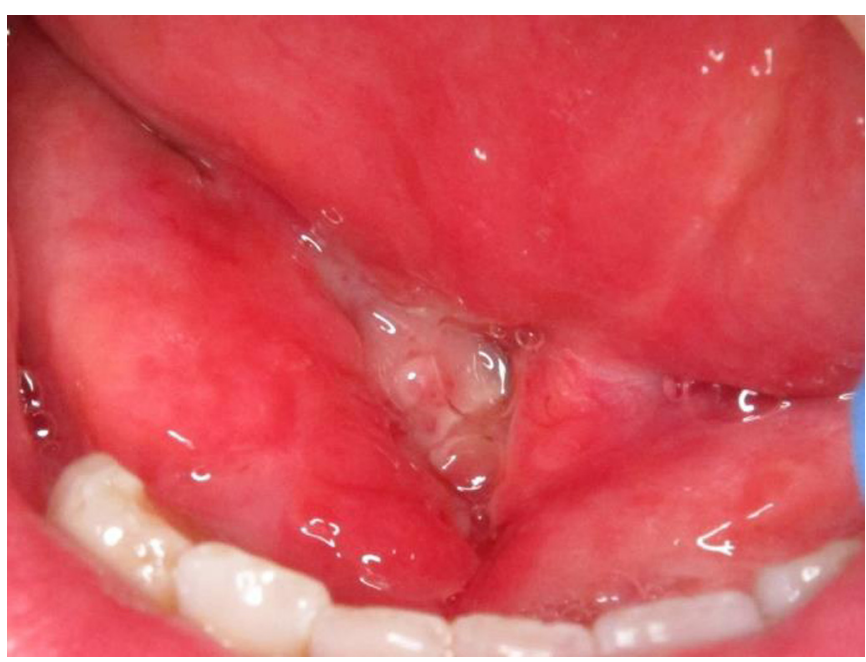

Fig. 2. Aspect of the lesion in November 2016 (intraoral photography).

The case presented is exceptional because of its chronic evolution. NS of the sublingual gland is the most likely diagnosis but could not be confirmed by the histopathological examination.

\section{Conflicts of interests}

The authors declare that they have no conflict of interest in relation to this article.

\section{References}

1. Abrams AM, Melrose RJ, Howell FV. Necrotizing sialometaplasia. A disease simulating malignancy. Cancer 1973;32:130-135.

2. Ledesma-Montes C, Garcés-Ortíz M, Salcido-García JF, HernándezFlores F. Review of the literature on necrotizing sialometaplasia and case presentation. Quintessence Int 2015;46:67-72.

3. Aubies-Trouilh S, Fricain JC. La sialométaplasie nécrosante : revue systématique de la littérature. Med Buccale Chir Buccale 2012;18:347-359.

4. Maisel RH, Johnston WH, Anderson HA, Cantrell RW. Necrotizing sialometaplasia involving the nasal cavity. Laryngoscope 1977;87:429-434. 